This item was submitted to Loughborough's Research Repository by the author.

Items in Figshare are protected by copyright, with all rights reserved, unless otherwise indicated.

\title{
Asymmetric internal solitary waves with a trapped core in deep fluids
}

PLEASE CITE THE PUBLISHED VERSION

LICENCE

CC BY-NC-ND 4.0

\section{REPOSITORY RECORD}

Derzho, Oleg G., and Roger H.J. Grimshaw. 2019. "Asymmetric Internal Solitary Waves with a Trapped Core in Deep Fluids". figshare. https://hdl.handle.net/2134/2712. 


\title{
Asymmetric Internal Solitary Waves with a Trapped Core in Deep Fluids
}

\author{
Oleg G. Derzho ${ }^{1}$ and Roger Grimshaw ${ }^{2}$ \\ ${ }^{1}$ Institute of Thermophysics, Russian Academy of Sciences, \\ 1 Lavrentyev Ave., Novosibirsk 630090, Russia \\ 2 Department of Mathematical Sciences, Loughborough University, \\ LE11 3TU, Leics, UK
}

February 9, 2007

\begin{abstract}
We describe an asymptotic model for long large-amplitude internal solitary waves with a trapped core, propagating in a narrow layer of nearly uniformly stratified fluid embedded in an infinitely deep homogeneous fluid. We consider the case of a mode one asymmetric wave with an amplitude slightly greater than the critical amplitude, for which there is incipient over-turning, that is wave-breaking. We then incorporate a vortex core located near the point at which this incipient breaking occurs. The effect of the vortex core is to introduce into the governing equation for the wave amplitude an extra nonlinear term proportional to the $3 / 2$ power of the difference between the wave amplitude and the critical amplitude. Thus the derived new equation for the wave amplitude incorporates both the nonlinearity arising due to the flow over the recirculation core, and the nonlinearity associated with the ambient stratification; the dispersion term however remains of the Benjamin-Ono type. We find that as the wave amplitude increases above the critical amplitude, the wave broadens, which is in marked contrast to the case of small amplitude waves where a sharpening of the wave crest normally occurs. The limiting form of the broadening wave is "a deep fluid bore". The wave speed is found to depend nonlinearly on the wave amplitude and the traditional linear dependence underestimates this speed.
\end{abstract}

\section{Introduction}

Solitary waves of permanent form propagating in a stratified fluid owe their existence to a balance between nonlinear wave steepening and linear wave dispersion. Recent reviews of these internal solitary waves in an oceanic context have been given by [1] and [2]. For waves of small but finite amplitude the nonlinearity is usually quadratic, and the coefficient 
in front of this quadratic term is determined by the specific profile of the stratification. Higher order expansions in the wave amplitude may account for higher-order nonlinearities [3], however, such an approach is limited to waves that do not overturn. Overturning occurs at a certain finite critical wave amplitude at which the horizontal velocity approaches zero in a frame of reference moving with the wave, that is, a local critical point appears inside the flow. Above this critical amplitude the critical point evolves into a vortex core that moves with the wave. In this paper we describe a model for long large-amplitude internal solitary waves with a vortex core, propagating in a narrow layer of nearly uniformly stratified fluid embedded in an infinitely deep homogeneous fluid. This study is an extension of the previous analytical work by [4] and [5] dealing with finite-amplitude waves in a similar geometry, but without any incipient overturning. A key observation that enables us to deal analytically with finite-amplitude waves is that, for the case of nearly uniform stratification in the layer, the nonlinearity is weak even if the wave amplitude is large. In fact, all non-overturning waves in the uniformly stratified environment are linear in the Boussinesq approximation. Thus the asymptotic procedure that uses an expansion parameter characterizing the deviation of stratification from the uniform profile does not require smallness of the wave amplitude. For waves with amplitudes slightly exceeding the critical amplitude, a further theoretical advance is possible. The shallow fluid case was studied in [6] and [7].

In this paper, we present a theoretical description of waves with a trapped core, propagating in a stratified layer embedded in deep fluid. The paper is organized as follows. In Section 2 we formulate the equations of motion and identify the relevant parameters. In Section 3 we present the derivation of an asymptotic long-wave equation describing a large amplitude steady wave with a small trapped core. Then we present numerical solutions of the derived equation for a particular profile of the stratification. In Sec 4 some concluding remarks will be given.

\section{Formulation}

Let us consider the two-dimensional steady motion of an ideal fluid. It is well known that in such system the density is constant along a streamline, and the vorticity equation yields a single equation for the streamfunction $([8])$,

$$
\begin{gathered}
\rho=\rho(\psi) \\
\psi_{x x}+\psi_{z z}+\frac{1}{\rho} \frac{d \rho}{d \psi}\left(g z+\frac{\psi_{x}^{2}+\psi_{z}^{2}}{2}\right)=G(\psi)
\end{gathered}
$$

where the $z$-axis is vertical upwards, the $x$-axis is horizontal, and $\psi$ is the streamfunction defined so that $u=-\psi_{z}$ and $v=\psi_{x}$, where $u$ and $v$ are the horizontal and vertical velocity components, respectively, and $\rho$ denotes the density. Subscripts here denote differentiation with respect to the appropriate variable. The functional forms of $\rho(\psi)$ and $G(\psi)$ are to be determined from the upstream conditions for those streamlines which originate upstream. 
Inside the vortex core, which is a zone with closed streamlines, the functions $\rho(\psi)$ and $G(\psi)$ will be determined in the sequel. Assuming that far upstream

$$
\psi \rightarrow c z \text { and } \rho \rightarrow \rho_{0}(z) \text { as } x \rightarrow \infty
$$

we can determine that

$$
\rho(\psi)=\rho_{0}(\psi / c) \text { and } G(\psi)=\frac{1}{\rho} \frac{d \rho}{d \psi}\left(\frac{g \psi}{c}+\frac{c^{2}}{2}\right)
$$

We consider the problem where a layer of stratified fluid is embedded between two infinitely deep homogeneous layers. It is convenient to introduce dimensionless (primed) variables by writing

$$
\psi^{\prime}=\frac{2 \psi}{c d}, x^{\prime}=\frac{2 x}{d}, z^{\prime}=\frac{2 z}{d} .
$$

where $d$ is the undisturbed thickness of the stratified layer. From now on we will operate with dimensionless variables and omit the prime superscript for simplicity. Further we assume that ambient stratification is weak, and only slightly deviates from a linear profile,

$$
\rho_{0}(z)=\rho_{00}(1-\sigma z-\sigma \theta S(z)), \sigma<<1, \quad \theta<<1, S \sim 1
$$

Here $\sigma<<1$ is the governing small parameter, and $\theta<<1$ is another small parameter whose size will be determined in the sequel. Thus the vorticity equation (2) becomes

$$
\begin{gathered}
\psi_{x x}+\psi_{z z}+\lambda(\psi-z)+\lambda \sigma(\psi-z) \psi-\frac{\sigma}{2}\left(\psi_{x}^{2}+\psi_{z}^{2}-1\right)+\theta \lambda(\psi-z) S_{\psi}=o(\sigma, \theta) \\
\text { where } \lambda=\frac{\sigma g d}{2 c^{2}} \sim 1 .
\end{gathered}
$$

Outside the stratified layer, the fluid is assumed to be homogeneous, so that

$$
\begin{aligned}
& \psi_{+x x}+\psi_{+z z}=0, \quad z>\eta_{+} \\
& \psi_{-x x}+\psi_{-z z}=0, \quad z \gtrless \eta_{-}
\end{aligned}
$$

where $\psi_{+}$and $\psi_{-}$denote the streamfunction in the upper and the lower half planes respectively, and $\eta_{+}$and $\eta_{-}$are the upper and the lower boundaries of the stratified layer. Assuming that the density is continuous across the boundaries of the stratified layer and that the waves are long compared with its thickness, the kinematic and dynamic boundary conditions at the boundaries of the stratified layer are the continuity of $\psi$ and $\psi_{z}$ across these boundaries. Indeed, the boundaries $z=\eta_{ \pm}$are streamlines, on which $\psi= \pm 1$ respectively. At infinity (as $x \rightarrow \pm \infty$ ), $\eta_{ \pm} \rightarrow \pm 1$, and $\psi \rightarrow z$.

If there is no recirculation zone inside the stratified layer, the set (7), (9), (10), and the boundary conditions provide a well-posed formulation of the problem. In this case the 
solutions must satisfy the condition that all streamlines originate upstream, which is the case when $\psi_{z}>0$ everywhere. This means that the horizontal velocity in the frame of reference moving with the wave is negative everywhere. It also ensures that stratification remains statically stable. This problem was solved by [4] and [5]. Here we consider the case when the afore-mentioned condition is violated, which we refer to as wave breaking. The critical condition, $\psi_{z}=0$, defines a critical wave amplitude and for waves with amplitudes greater than this critical amplitude, there is a vortex core containing recirculating fluid inside the stratified layer. Outside the recirculation zone, the flow is defined by (7), (9), (10), the boundary conditions at infinity, and also by the conditions of continuity of $\psi$ and $\psi_{z}$ on the boundaries $\eta_{R \pm}$ of the recirculation zone. These simplified matching conditions are valid for the long-wave limit considered here. Inside the recirculation zone we assume that the fluid has a constant density equal to that on the recirculation zone boundary, which is a streamline. Thus inside the recirculation zone

$$
\psi_{x x}+\psi_{z z}=H(\psi)
$$

where $H(\psi)$ is yet to be determined. The region where there is a recirculation zone is referred to as the inner zone, and the remaining region is then referred to as the outer zone.

\section{Derivation of the amplitude equation}

We seek solitary wave solutions whose width is much greater than the thickness of the stratified layer. Hence we introduce the small parameter $\varepsilon$ and let

$$
X=\varepsilon x
$$

be the new horizontal variable. Inside the stratified layer we then seek a solution in the form of the asymptotic expansions

$$
\begin{gathered}
\psi(X, z)=\psi^{(0)}(X, z)+\varepsilon \psi^{(1)}(X, z)+\ldots \\
\lambda=\lambda^{(0)}+\varepsilon \lambda^{(1)}+\ldots \\
\eta_{ \pm}=\eta_{ \pm}^{(0)}+\varepsilon \eta_{ \pm}^{(1)}+\ldots
\end{gathered}
$$

Outside the stratified layer there are no physical grounds to assume that the vertical scale is much smaller than the horizontal one, so we introduce stretched vertical coordinates,

$$
Z_{ \pm}=\varepsilon z
$$

Then we seek solutions of the governing equations outside the stratified layer in the following form, 


$$
\begin{gathered}
\psi_{+}=z+\phi_{+}\left(X, Z_{+}\right), \quad Z_{+}>\varepsilon \eta_{+} \\
\psi_{-}=z+\phi_{-}\left(X, Z_{-}\right), \quad Z_{-} \gtrless \varepsilon \eta_{+} \\
\left(\frac{\partial^{2}}{\partial X^{2}}+\frac{\partial^{2}}{\partial Z_{ \pm}^{2}}\right) \phi_{ \pm}=0 \\
\phi_{ \pm}\left(X, Z_{ \pm}\right) \rightarrow 0 \text { as } X^{2}+Z_{ \pm}^{2} \rightarrow \infty
\end{gathered}
$$

The solution of (17), (18), (19), (20) with a Dirichlet boundary condition can be found in the form,

$$
\phi_{ \pm}\left(X, Z_{ \pm}\right)= \pm \frac{1}{\pi} \int_{-\infty}^{\infty} \frac{Z_{ \pm} A_{ \pm}\left(X^{\prime}\right)}{\left(X-X^{\prime}\right)^{2}+Z_{ \pm}^{2}} d X^{\prime}
$$

where $A_{ \pm}(X)=\phi_{ \pm}(X, 0)$. In the vicinity of the stratified layer,we find from (21) that

$$
\psi_{ \pm}^{(0)}=z+A_{ \pm}(X), \quad \psi_{ \pm}^{(1)}=\mp \frac{z}{\pi} \frac{\partial}{\partial X} \int_{-\infty}^{\infty} \frac{A_{ \pm}\left(X^{\prime}\right)}{X-X^{\prime}} d X^{\prime}
$$

Inside the stratified layer, we must solve (7). together with the boundary conditions at $z=\eta_{ \pm}$, which are that $\psi= \pm 1$ on these boundaries and that $\psi_{z}$ is continuous there. At the leading order, we see from $(22)$ that $\psi_{z}^{(0)}=0$ on $z=\eta_{ \pm}^{(0)}$. Hence, we find that at the lowest order, $A_{ \pm}(X)=A(X)$ and that

$$
\begin{gathered}
\psi^{(0)}=z+(-1)^{n} A(X) \cos \left(\pi n(z+A(X)), \quad \lambda_{n}^{(0)}=(\pi n)^{2},\right. \\
\eta_{ \pm}^{(0)}= \pm 1-A(X)
\end{gathered}
$$

where $n=1,2,3, \cdots$ is an integer. Here $A(X)$ will be determined from the solvability condition for the first order equations. We will consider only the fastest wave $n=1$, as the higher modes can be treated similarly. Interaction between the modes is beyond the scope of the present study.

At the first order we obtain

$$
\psi_{z z}^{(1)}+\lambda^{(0)} \psi^{(1)}+F^{(1)}=0,
$$

$$
\begin{aligned}
& \text { where } \quad F^{(1)}=\lambda^{(1)}\left(\psi^{(0)}-z\right)-\frac{\sigma}{\varepsilon}\left(\lambda^{(0)}\left(\psi^{(0)}-z\right) \psi^{(0)}+\frac{\psi_{z}^{(0) 2}-1}{2}\right) \\
& +\frac{\theta}{\varepsilon} \lambda^{(0)}\left(\psi^{(0)}-z\right) S_{\psi}\left(\psi^{(0)}\right) \text {. }
\end{aligned}
$$


The expression for $F^{(1)}$ indicates that $\sigma$ and $\theta$ should both scale with $\varepsilon$ (see below). Equation (25) needs to be solved along with the matching conditions on the boundaries of the stratified layer. It is straightforward to show that

$$
\begin{gathered}
\psi_{z}^{(1)}\left(\eta_{+}^{(0)}\right)-\psi_{z}^{(1)}\left(\eta_{-}^{(0)}\right)+A \lambda^{(0)}\left(\psi^{(1)}\left(\eta_{+}^{(0)}\right)-\psi^{(1)}\left(\eta_{-}^{(0)}\right)\right)= \\
-\frac{2}{\pi} \frac{\partial}{\partial X} \int_{-\infty}^{\infty} \frac{A\left(X^{\prime}\right)}{X-X^{\prime}} d X^{\prime}
\end{gathered}
$$

Using (27) the solvability condition for the first order equation can be found by multiplying (25) by $A(X) \pi \sin (\pi(z+A(X)))-\cos (\pi(z+A(X)))$ and integrating over the stratified layer, to get

$$
\begin{gathered}
-\frac{2}{\pi} \frac{\partial}{\partial X} \int_{-\infty}^{\infty} \frac{A\left(X^{\prime}\right)}{X-X^{\prime}} d X^{\prime}-\lambda^{(1)} A+\frac{\sigma}{\varepsilon} \frac{3 \pi^{2}}{2} A^{2}+\frac{\theta}{\varepsilon} M(A)=0 \\
\text { where } \quad M(A)=-\pi^{2} \int_{-1}^{1} A S_{\psi}(Y) \cos (\pi y)(\cos (\pi y)-A \pi \sin (\pi y)) d y \\
\text { and } Y=y-A-\cos (\pi y)
\end{gathered}
$$

Equation (28) is a generalization of the well-known Benjamin-Ono equation ([9], [10]) derived in [4],[5]. The critical condition that $\psi_{z}=0$, when applied to the leading order term (23), shows that incipient overturning for a wave of depression (elevation) is reached at $z=-1 / 2-1 / \pi(z=1 / 2+1 / \pi)$ when the amplitude is $A_{*}=1 / \pi\left(A_{*}=-1 / \pi\right)$. Note that the critical point is located inside the stratified layer, and that the notation wave of elevation, or depression, applies to the behavior of the boundaries of the stratified layer, while some streamlines inside the layer may behave in the opposite manner compared to the boundaries of the stratified layer. Equation (28) holds in the whole stratified layer provided that $|A|<\left|A_{*}\right|$ everywhere, when there is no recirculation zone. It also holds when there is a recirculation zone, but is then confined to the outer region where $|A|<\left|A_{*}\right|$. But in this case it must be matched to an appropriate solution in the inner zone, which incorporates a recirculation core. This matching will now be described below.

We suppose that the recirculation zone occupies the region $\eta_{R_{-}}<z<\eta_{R+}$, and then define the inner zone as the region where $\eta_{R \pm} \neq 0$. Then, for the wave of depression $(A>0)$ we will show that

$$
\eta_{R \pm}=-1 / 2-1 / \pi \pm \eta_{R}
$$

We assume that the recirculation core has a long length scale and a small vertical extent, both when compared to the thickness of the stratified layer, so that we may put

$$
\eta_{R}=\delta f(\xi), \xi=\beta x, \delta<<1, \beta<<1 .
$$

We further suppose that the wave amplitude $A(\xi)$ in the inner zone is close to the critical amplitude $A_{*}=1 / \pi$, 


$$
A(\xi)=A_{*}+\mu B(\xi), \mu<<1 .
$$

The relationship between $\delta, \beta, \mu$ and $\varepsilon$ will be determined later. Outside the recirculation core we again seek a solution in the form (13), but now with the slow variable $\xi$. The zeroth order solution (23) and the first order equation (25) and the boundary conditions all still hold but now $F^{(1)}=F^{(1)}\left(A_{*}, z\right)$ and matching of the solutions inside and outside the recirculation zone needs to be done. The compatibility condition can be obtained similarly to $(28)$ but the integration is from $\eta_{-}^{(0)}$ to $\eta_{R-}$, and then from $\eta_{R+}$ to $\eta_{+}^{(0)}$. Thus we get

$$
\begin{aligned}
\psi_{z}^{(1)}\left(\eta_{R-}\right)-\psi_{z}^{(1)}\left(\eta_{R+}\right) & +\pi\left(\psi^{(1)}\left(\eta_{R-}\right)-\psi^{(1)}\left(\eta_{R+}\right)\right)-\frac{2}{\pi} \frac{\partial}{\partial X} \int_{-\infty}^{\infty} \frac{A\left(X^{\prime}\right)}{X-X^{\prime}} d X^{\prime} \\
= & -\lambda^{(1)} A_{*}+\frac{\sigma}{\varepsilon} \frac{3 \pi^{2}}{2} A_{*}^{2}+\frac{\theta}{\varepsilon} M\left(A_{*}\right)
\end{aligned}
$$

The Hilbert integral in (34) comprises three parts, an integration over the inner zone and integrations over the outer zone upstream and downstream of the inner zone. The integral over the inner zone should be of the same order (in the asymptotic sense). This leads to the relation

$$
\varepsilon=\beta \mu
$$

Next we turn to the recirculation core boundary conditions, in order to calculate the undetermined terms on the left-hand side of (34). Our key physical assumption is that the flow is stagnant inside the core, at least at the leading order. The reason for this is described later, when the relationship between the various small parameters is defined. For a stagnant core, the incipient overturning condition must hold along the core boundary, that is, $\psi_{z}\left(\eta_{R \pm}\right)=0$, which leads to

$$
f(\xi)=\sqrt{\frac{2}{\pi} \frac{\mu}{\delta^{2}} B(\xi)} .
$$

We see now that we should impose the balance $\mu=\delta^{2}$. It is now clear that the recirculation zone is symmetric, confirming the form of (31). The kinematic boundary condition across the border of the stagnant zone yields

$$
\varepsilon \psi^{(1)}\left(\eta_{R \pm}\right)-\mu B(1 \pm \pi \delta f) \pm \pi^{2}(\delta f)^{3} / 6=0
$$

Using (36), (37), (34) in the inner zone and (28) in the outer zone, we now can obtain the amplitude equation which is valid in the whole domain,

$$
\frac{2}{\pi} \frac{\partial}{\partial X} \int_{-\infty}^{\infty} \frac{A\left(X^{\prime}\right)}{X-X^{\prime}} d X^{\prime}=\left\{\begin{array}{c}
-\lambda^{(1)} A+\frac{\sigma}{\varepsilon} \frac{3 \pi^{2}}{2} A^{2}+\frac{\theta}{\varepsilon} M(A) \text { if }|A|<\left|A_{*}\right| \\
-\lambda^{(1)} A_{*}+\frac{\sigma}{\varepsilon} \frac{3 \pi^{2}}{2} A_{*}^{2}+\frac{\theta}{\varepsilon} M\left(A_{*}\right)-\frac{(2 \pi)^{3 / 2}}{3} \frac{\mu^{3 / 2}}{\varepsilon} B^{3 / 2} \\
\text { if }|A|>\left|A_{*}\right|
\end{array}\right.
$$


The requirement that all terms on the right-hand side of (38) are of the same order leads to the scaling

$$
\varepsilon=\mu^{3 / 2}, \beta=\mu^{1 / 2}, \delta=\mu^{1 / 2}, \frac{\sigma}{\varepsilon} \sim 1, \frac{\theta}{\varepsilon} \sim 1 .
$$

We have assumed that the flow inside the recirculation zone is stagnant in the frame of reference moving with the wave. This can be justified in the following way. First we argue that the vorticity inside the recirculation zone and at the vortex core boundary should be of the same order with respect to the governing small parameter. Next we recall that the fluid density inside the core is constant, and equal to the density at its boundary. Then it is readily shown that just outside the core the vorticity is $O(\delta)$. Thus inside the core we replace (11) with

$$
\beta^{2} \psi_{\xi \xi}+\psi_{z z}=\delta H(\psi)
$$

This equation is to be solved with the boundary conditions that require continuity of $\psi$ and $\psi_{z}$ across the boundaries of the core. Thus, in particular,

$$
\psi=-1 / 2-1 / \pi \text { on } z=-1 / 2-1 / \pi \pm \delta f
$$

Let us rescale the sreamfunction and the vertical coordinate inside the core as follows

$$
\begin{aligned}
& \psi=-1 / 2-1 / \pi+\delta \Phi \\
& z=-1 / 2-1 / \pi+\delta Z
\end{aligned}
$$

Using (42),(39) and (43) we rewrite (40) as

$$
\begin{aligned}
& \Phi_{Z Z}=\delta^{2} \Omega+O\left(\delta^{3}\right), \\
& \Phi=0 \text { on } Z= \pm f,
\end{aligned}
$$

where $\Omega=H(0)$ is a constant. The solution of (44) and (45) is

$$
\Phi(\xi, Z)=\delta^{2} \frac{\Omega}{2}\left(Z^{2}-f^{2}\right)+O\left(\delta^{3}\right) .
$$

It now follows that $\Phi_{Z}(\xi, \pm f)= \pm \delta^{2} \Omega f$ and this can only be matched with $\psi_{z}\left(\eta_{R \pm}\right)=O(\varepsilon)$ from the inner flow if $\Omega=0$; thus from (46) we see that the flow inside the core is indeed stagnant at the leading order.

\section{Analysis of the amplitude equation}

Now we turn to the analysis of the amplitude equation (38). It is interesting to note that the nonlinearity $\left(A-A_{*}\right)^{3 / 2}$ arising due to the flow over the stagnant core does not depend on the details of stratification. Solutions of the equation (38) can be found numerically, 
and the results of our calculations are presented below. Here, for simplicity, we present results for only one particular case

$$
S(z)=-3 / \pi^{2}-3 / 2, \varepsilon=\sigma=\theta=0.02,
$$

in the expression for the undisturbed stratification (6). In this case, the nonlinearity due to the effects of stratification reduces to just

$$
\frac{\sigma}{\varepsilon} \frac{3 \pi^{2}}{2} A^{2}+\frac{\theta}{\varepsilon} M(A)=-\frac{3}{2} A^{2} .
$$

Thus when $|A|<\left|A_{*}\right|$ the wave amplitude obeys the standard Benjamin-Ono equation with quadratic nonlinearity [9],[10]. Solitary wave solutions of that equation are the wellknown algebraic solitons. However we recall that here the wave amplitude is not small and for the stratification (47) the Benjamin-Ono equation is valid up to the incipient breaking point at $A=A_{*}$. For this special case (47) an internal solitary wave propagating inside the stratified layer leads to a local depression of its boundaries. In Fig.1 we show the streamline structure for a wave with a recirculation core for $A_{\max }=1.01584 A_{*}$. A zone with closed streamlines is clearly seen inside the stratified layer.

In Fig.2 we show the width of the recirculation zone as a function of the normalized wave amplitude $A / A_{*}$. The width of the wave at the crest increases as the wave amplitude increases. However, there is a maximum possible amplitude at which the wave width becomes infinite, that is, the solitary wave turns into a bore. This maximum amplitude is quite close to the critical amplitude $A_{*}$ thus confirming our theoretical assumption (33).

Concerning the wave speed the functional form of (38) indicates that its dependence on the amplitude is nonlinear which is in contrast to the linear dependence for the standard Benjamin-Ono equation. The formula for the wave speed is

$$
c=\frac{\sqrt{\sigma g d / 2}}{\pi}\left(1-\varepsilon \frac{\lambda^{(1)}}{2 \pi^{2}}\right)
$$

In Fig. 3 we show $\lambda^{(1)}$ as a function of the normalized wave amplitude $A / A_{*}$. It is seen that the traditional linear dependence underestimates the wave speed; a similar result was analytically predicted in [3] for the mode one (according to the terminology of [3]) internal wave propagating in a narrow stratified layer confined within a deep fluid.

In Fig.4 we show the streamline pattern in the entire stratified layer for $A_{\max }=$ $1.01584 A_{*}$. The upper plot corresponds to calculations based on the presented model, while the lower plot shows the predictions of the traditional Benjamin-Ono model for a wave with the same amplitude. It is readily seen that the Benjamin-Ono model is unable to predict the structure of the large amplitude wave with a trapped core.

Although the linear dispersive term on the left-hand side of the amplitude equation (38) is a nonlocal Hilbert integral, the rate of decay of the wave as $X \rightarrow \pm \infty$ can be determined locally by balancing the linear terms in the far-field. For this purpose, we represent the left-hand side of (38) in terms of the Fourier transform of $A$ as follows 


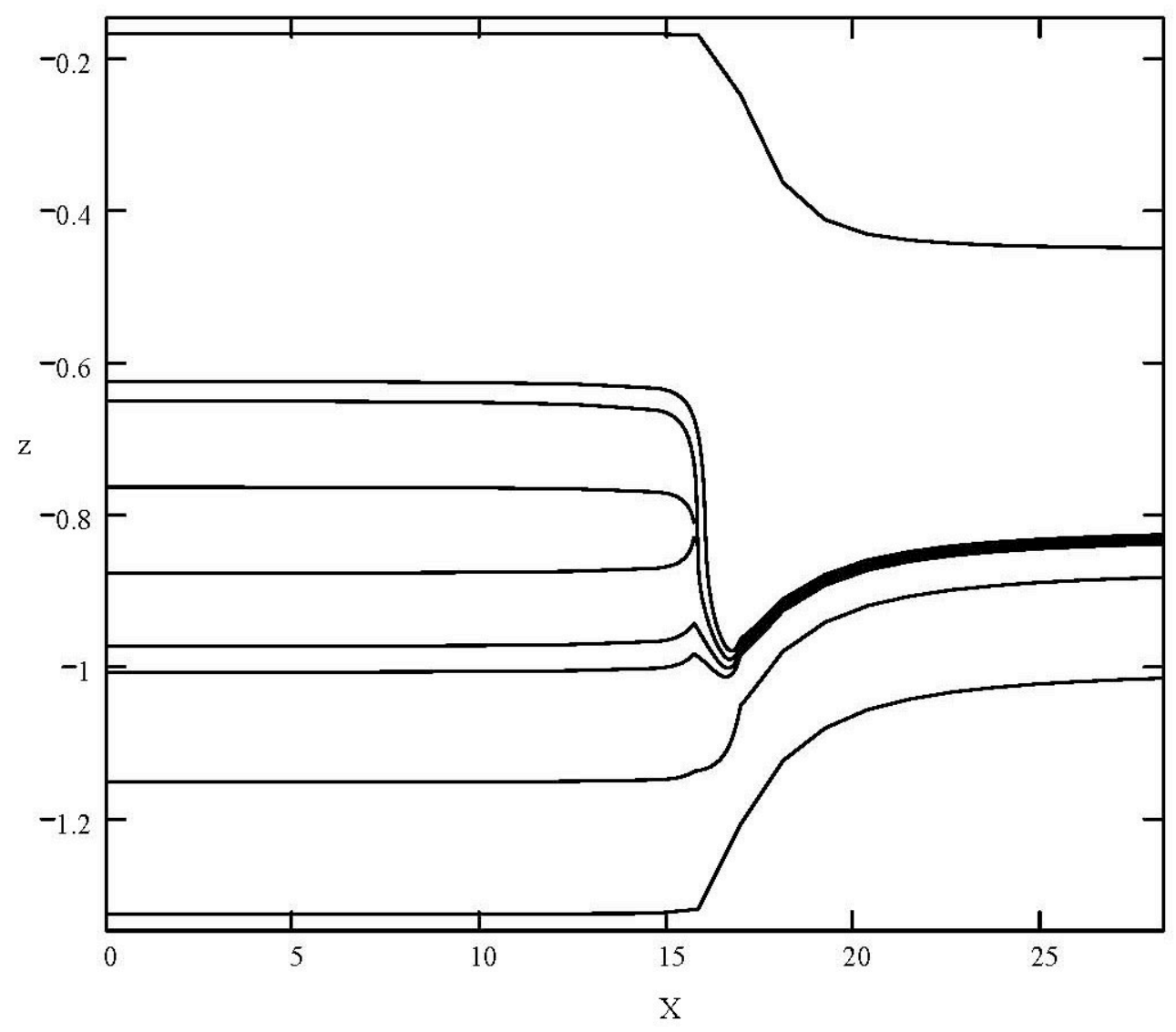

Figure 1: Streamline pattern. 


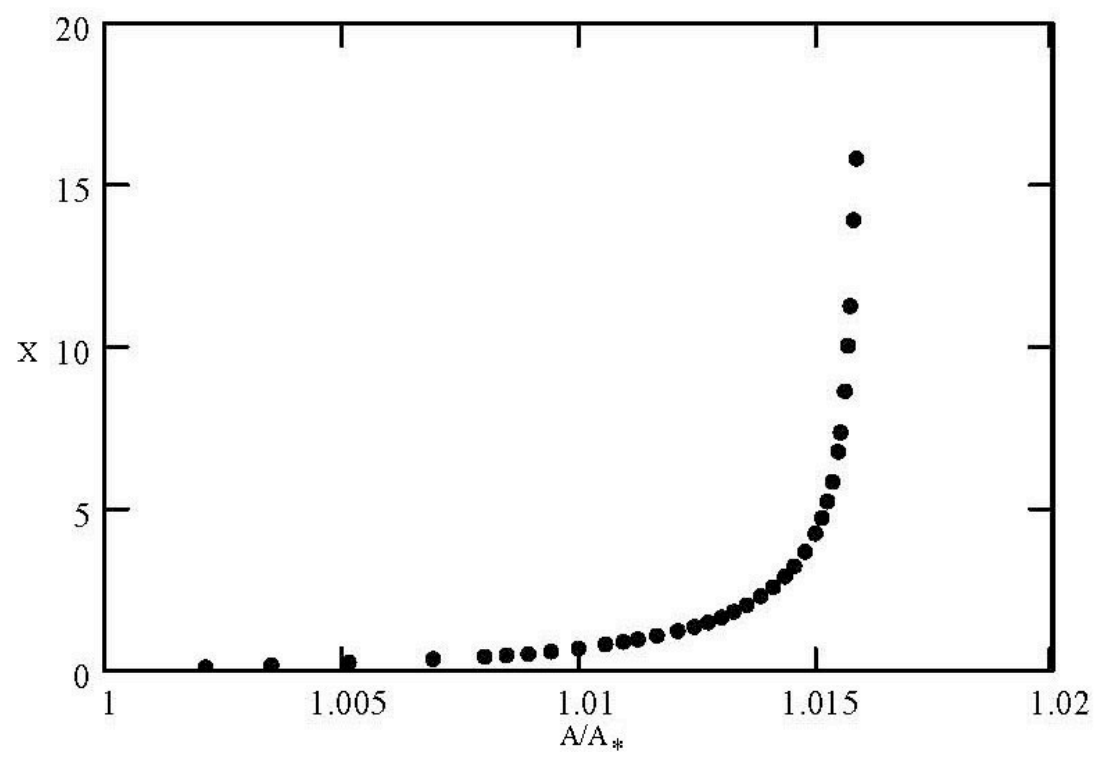

Figure 2: Width of the stagnation core as a function of the normalized wave amplitude $A / A_{*}$.

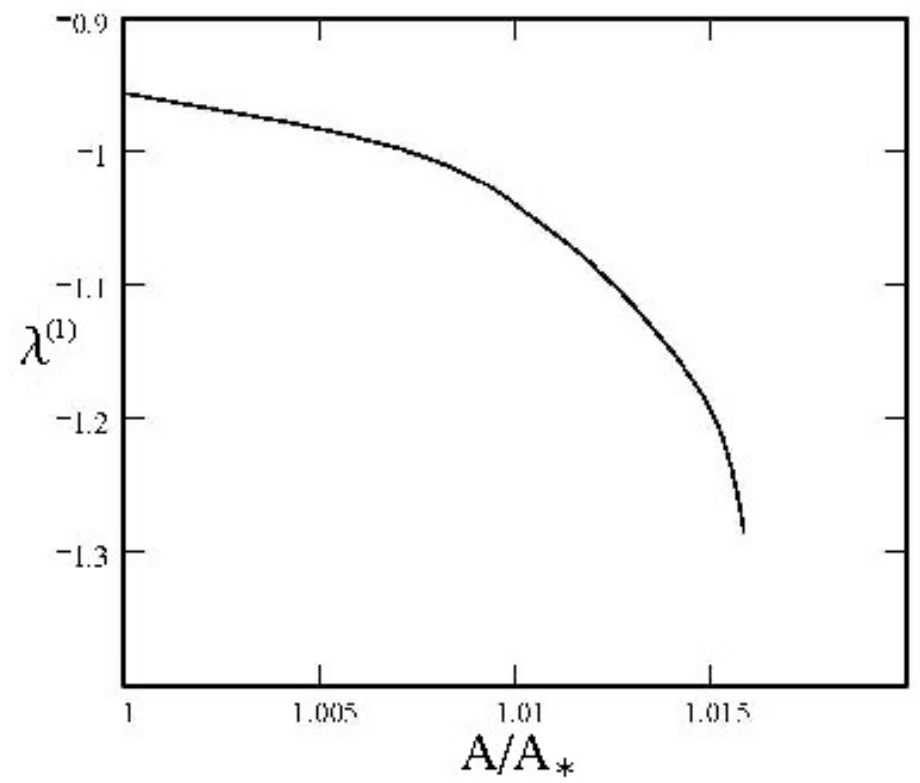

Figure 3: $\lambda^{(1)}$ as a function of the normalized wave amplitude $A / A_{*}$. 

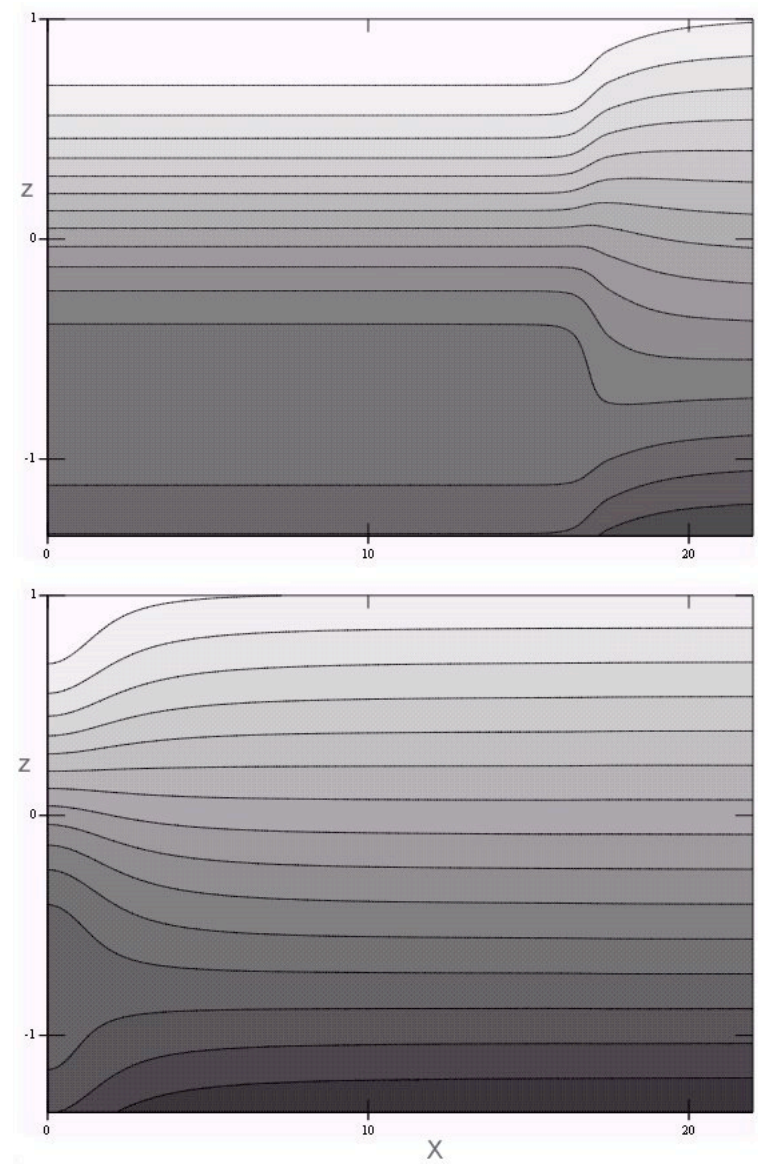

Figure 4: Streamline pattern in the entire stratified layer. Upper plot correspons to the calculations using equation (38), while the lower plot shows what the traditional BenjaminOno equation would predict for the same wave amplitude. 


$$
\begin{aligned}
\frac{2}{\pi} \frac{\partial}{\partial X} \int_{-\infty}^{\infty} \frac{A\left(X^{\prime}\right)}{X-X^{\prime}} d X^{\prime} & =\frac{1}{\pi} \int_{-\infty}^{\infty}|k| \hat{A}(k) \exp (i k X) d k \\
\text { where } \hat{A}(k) & =\int_{-\infty}^{\infty} A(X) \exp (-i k X) d X
\end{aligned}
$$

We can now evaluate the right-hand side of (50) as $X \rightarrow \pm \infty$ by the standard method of integrating by parts. The outcome is

$$
\frac{2}{\pi} \frac{\partial}{\partial X} \int_{-\infty}^{\infty} \frac{A\left(X^{\prime}\right)}{X-X^{\prime}} d X^{\prime}=-\frac{2 \hat{A}(0)}{\pi X^{2}}+O\left(\frac{1}{X^{4}}\right) \quad \text { as } X \rightarrow \pm \infty
$$

Here we have used the symmetry of $A(X)=A(-X)$. Substitution into (38) now gives

$$
A(X)=\frac{2 \hat{A}(0)}{\pi \lambda^{(1)} X^{2}}+O\left(\frac{1}{X^{4}}\right) \quad \text { as } X \rightarrow \pm \infty .
$$

But note that as the wave amplitude aproaches the maximum amplitude, the region where this $1 / X^{2}$ asymptotic behaviour is valid will move further away from the wave crest as the wave broadens.

\section{Conclusion}

We have presented a model for internal solitary waves propagating in a thin stratified layer embedded in an infinite fluid of constant density. The stratification is assumed to be weak and nearly uniform. The main result is that a new equation for the wave amplitude is derived which accounts for waves with amplitudes beyond the critical amplitude at which the incipient overturning occurs, and a vortex core appears inside the wave. The effect of the vortex core is to introduce into the amplitude equation an extra nonlinear term proportional to the $3 / 2$ power of the difference between the wave amplitude and the critical amplitude. Thus the derived equation incorporates the nonlinearity arising due to the flow over the recirculation core, and the nonlinearity associated with the ambient stratification; the dispersion term, however, remains of the Benjamin-Ono integral type. We note that the form of the extra term does not depend on the details of the stratification. We find that as wave amplitude increases above the critical amplitude, the wave broadens, which is in marked contrast to the case of small amplitude waves where sharpening of the wave crest normally occurs. A similar effect was recently reported in the numerical calculations of [11] although for a different flow geometry. Further, the wave speed is found to depend nonlinearly on the wave amplitude. and the traditional linear dependence underestimates this speed. The limiting form of the broadening wave is a deep fluid bore. 


\section{Acknowledgments}

OD acknowledges the hospitality offered by the Department of Mathematical Sciences, Loughborough University where part of this work was carried out. The work was supported by the Siberian Division of the Russian Academy of Sciences - EU INTAS paternship grant N 06-1000013-9236.

\section{References}

[1] K. R. Helfrich and W.K. Melville, Annual Rev. Fluid Mechanics 38,395-425 (2006)

[2] E. N. Pelinovsky, O. Polukhima, A. Slunyaev and T. Talipova, Internal Solitary Waves, in "Solitary Waves in Fluids", ed. R. Grimshaw, WIT Press, Ch. 4 , 85-110, (2007).

[3] R. Grimshaw, Phys. Fluids, 24, 1611-1618 (1981)

[4] D. J. Benney and R. Grimshaw, Stud. Appl. Math., 66, 181-187 (1982).

[5] O.G. Derzho O G and M.G. Velarde, Phys. Fluids, 7(6), 1357-1362 (1995).

[6] O.G. Derzho and R. Grimshaw, Phys, Fluids, 9(11), 3378-3385 (1997).

[7] O.G. Derzho and R. Grimshaw, Stud. Appl. Math., 115,387-403 (2005).

[8] M.L. Dubreil-Jacotin, Atti. Reale Acad. Nationale Lincei, 15(6),44-52 (1932).

[9] T. B. Benjamin, J. Fluid Mech., 29,559-592 (1967).

[10] H. Ono, J. Phys. Soc. Japan, 39,1082-1091 (1975).

[11] K.G. Lamb and K. Wilkie, Phys. Fluids, 16, 4685-4695 (2004) 